\title{
DIFERENTES ÉPOCAS DE COLHEITA, SECAGEM E ARMAZENAMENTO NA QUALIDADE DE GRÃOS DE TRIGO COMUM E DURO ${ }^{(1)}$
}

\author{
LUCIANA MARIA TERRA ALVES CARNEIRO $^{(2)}$; JOÃO DOMINGOS BIAGI ${ }^{(2)}$; \\ JOSÉ GUILHERME DE FREITAS ${ }^{(3)}$; MARCELO CRISTIANO CARNEIRO ${ }^{(4)}$; JOÃO CARLOS FELÍCIO(3)
}

\section{RESUMO}

O objetivo do trabalho foi verificar a influência da época de colheita, secagem e período de armazenamento na qualidade de grãos de trigo comum e duro. Os experimentos foram instalados no campo do Núcleo Experimental de Campinas, IAC, usando DOIS genótipos de trigo comum (Triticum aestivum L), um com dormência na espiga (IAC 24), colhido com 30,0\%; 21,4\% e 12,2\% de água e um sem dormência na espiga (IAC 289), colhido com 35,0\%; 23,4\% e 12,5\% de água; além de um genótipo de trigo duro (Triticum durum L.) sem dormência (IAC 1003), colhido com 31,6\%; 22,2\% e 11,7\% de água. A secagem foi realizada a 40,60 e $80{ }^{\circ} \mathrm{C}$ e um fluxo de ar de $20 \mathrm{~m}^{3} \mathrm{~min}^{-1} \cdot \mathrm{m}^{-2}$. Após a secagem, os grãos foram armazenados em embalagens de polietileno por um período de $0,2,4,6$ e 8 meses a $20 \pm 2{ }^{\circ} \mathrm{C}$. Os parâmetros de qualidade de grãos analisados no Laboratório de cereais, raízes e tubérculos na FEA/ UNICAMP foram os testes de número de queda, viscosidade, força geral do glúten e peso hectolitro. Observou-se no genótipo IAC 289 um aumento significativo da atividade da enzima alfa-amilase, com o atraso da colheita. Com antecipação da colheita e da secagem não foi reduzida a qualidade dos grãos e, com a armazenagem, houve melhora na qualidade tecnológica da farinha do trigo.

Palavras-chave: alfa-amilase, Triticum aestivum, Triticum durum.

\section{ABSTRACT \\ HARVEST TIMING, DRYING AND STORAGE EFFECTS IN THE GRAIN QUALITY OF COMMON AND HARD WHEAT}

The objective of the present work was to analyse the effects of initial moisture content, drying parameters and storage period in grain quality. The experiment was installed in Campinas Agronomic Institute (IAC), using two genotypes of common wheat (Triticum aestivum L), one with spike dormancy (IAC 24), harvested with $30.0 \% ; 21.4 \%$ and $12.2 \%$ moisture content and the other without it (IAC 289), harvested with $35.0 \% ; 23.4 \%$ and $12.5 \%$ moisture content; additionally, one hard wheat genotype (Triticum durum L.) without dormancy (IAC 1003), harvested with $31.6 \%, 22.2 \%$ and $11.7 \%$ moisture content was also analysed. Drying operations were performed at 40,60 and $80{ }^{\circ} \mathrm{C}$ under an air flow of $20 \mathrm{~m}^{3} \mathrm{~min}^{-1} \cdot \mathrm{m}^{-2}$. Dried grains were then stored in polyethylene packets for a period of $0,2,4,6$ and 8-months at $20 \pm 2^{\circ} \mathrm{C}$. Grain quality parameters analysed were number of falls, viscosity, general force of gluten and hectolitre weight. The genotype IAC 289 presented an increase on alpha-amylase activity due to harvest delay. Harvest anticipation and drying process had no effect on grain quality, whereas storage period improved the technological quality of the wheat flour.

Key words: alpha-amylase, Triticum aestivum, Triticum durum.

( $\left.{ }^{1}\right)$ Recebido para publicação em 2 de outubro de 2003 e aceito em 8 de novembro de 2004.

$\left({ }^{2}\right)$ Faculdade de Engenharia Agrícola, UNICAMP, Caixa Postal 6011, 13080-970 Campinas (SP). E-mail: lualves@agr.unicamp.br; biagi@agr.unicamp.br

( ${ }^{3}$ ) Centro de Análise e Pesquisa Tecnológica do Agronegócio de Grãos e Fibras, IAC, Caixa Postal 28, 13001-970 Campinas (SP).

$\left({ }^{4}\right)$ Doutorando em Tecnologia Pós-colheita (FEAGRI/UNICAMP). E-mail: carneiro@agr.unicamp.br 


\section{INTRODUÇÃO}

O trigo é de grande importância para a economia do Brasil, devido ao elevado consumo de seus derivados, principalmente pão, macarrão e farinha. No entanto, a produção nacional do grão não tem sido suficiente para atender a demanda, agravada pela grande quantidade de grãos perdidos ou colhidos com qualidade inferior, decorrentes de ataque de insetos ou ocorrência de chuvas no período da colheita.

A produção brasileira de trigo em 2002 foi de aproximadamente 3,20 milhões de toneladas, praticamente igual à de 2001 (3,26 milhões de toneladas), sendo frustradas as expectativas, pois houve perda de 400 mil toneladas, em relação à previsão inicial, devido às adversidades climáticas. Como conseqüência, foram importados 7,4 milhões de toneladas, para atendimento de demanda de 10,6 milhões de toneladas (FNP, 2002).

Há cultivares de trigo comum e duro que não apresentam dormência, podendo germinar na espiga antes do processo de colheita, se houver precipitação pluvial, o que pode inviabilizar o uso dos grãos pela indústria alimentícia e a produção de sementes.

Nas regiões produtoras de trigo do Brasil, durante o período da colheita, é comum a ocorrência de chuvas, acarretando elevada incidência de grãos germinados, classificados como de qualidade inferior para a indústria panificadora, com depreciação de seu valor ao redor de $50 \%$. Considerando-se que a cada ano cerca de $20 \%$ da safra de trigo é de grãos germinados, obter-se-iam aproximadamente $437 \mathrm{mil}$ toneladas de trigo germinado (BRUM, 2000).

O processo de antecipação da colheita em genótipos de trigo sensíveis à germinação na espiga pode ser uma alternativa para se obter um produto de boa qualidade industrial ou para a produção de grãos, viabilizando-se o cultivo desses genótipos para colheita com a umidade do trigo acima da normal $13 \%$ a $15 \%$. Após a fertilização, o tamanho da semente ou grão aumenta gradativamente até atingir o peso da matéria seca, quando a semente não mais recebe fotossintetizados da planta e pode ser considerada como "desligada" da planta mãe (CARvalho e NAKAGAWA, 2000) Nesse ponto, tem-se a maturidade fisiológica da semente, quando o conteúdo de reservas é máximo e o teor de água ainda é muito alto - entre $30 \%$ e 50\%, dependendo da espécie (Delouche, 1971; Harrington, 1972; Toledo e Marcos Filho, 1977; Carvalho e Nakagawa, 2000).

A maioria dos produtos agrícolas, como grãos, sementes e frutos, segundo Martins et al. (1999), deveria ser colhida no ponto de maturação fisiológica, quando estão com teores elevados de amido, proteínas e água. Entretanto, em produtos com elevada umidade há atividade metabólica mais intensa, sendo propício o desenvolvimento de fungos e insetos e deterioração rápida. Por meio da remoção de umidade pela secagem e de um correto armazenamento, torna-se possível a conservação de produtos agrícolas. Com a operação de secagem é assegurada maior qualidade dos grãos, por possibilitar colheitas antecipadas, evitando-se danos no campo devido às condições climáticas, ataques de insetos e microrganismos.

Assim, o objetivo do trabalho foi avaliar a influência da antecipação da colheita, da secagem artificial e do armazenamento na qualidade de grãos de Triticum durum L. e Triticum aestivum $\mathrm{L}$.

\section{MATERIAL E MÉTODOS}

O experimento no campo foi instalado em junho de 2001, no Centro Experimental Central do Instituto Agronômico, em Campinas, em Latossolo Vermelho, com três repetições, usando dois genótipos de trigo comum (Triticum aestivum $\mathrm{L}$ ), um com dormência na espiga - IAC 24 - colhido com $30,0 \%$ na $1 .^{\text {a }}$ colheita, $21,4 \%$ na $2 .^{a}$ e $12,2 \%$ de água na $3 .^{\text {a }}$; um sem dormência na espiga - IAC 289 - colhido com $35,0 \%$ na $1 .^{\mathrm{a}}$ colheita, 23,4 na $2 .^{\mathrm{a}}$ e $12,5 \%$ de água na 3. ${ }^{\text {a }}$; um genótipo de trigo duro (Triticum durum L.) sem dormência - IAC 1003 - colhido com $31,6 \%$ na 1 . $^{\mathrm{a}}$ colheita, com $22,2 \%$ na $2 .^{a}$ colheita e com $11,7 \%$ de água na $3 .^{\mathrm{a}}$ colheita. Foram retiradas amostras de trigo de acordo com a época de colheita, e enviadas ao Laboratório da Faculdade de Engenharia Agrícola - UNICAMP, para os testes de secagem e qualidade de grãos.

Após a colheita, os grãos foram separados em amostras para serem submetidas aos testes de secagem, apenas para a $1 .^{\mathrm{a}}$ e a $2 .^{\mathrm{a}}$ colheitas, realizados no laboratório de secagem da Faculdade de Engenharia Agrícola da UNICAMP. Para cada tratamento de secagem foram usados aproximadamente $15 \mathrm{~kg}$ de produto.

Foi utilizado um secador de madeira de base quadrada com sistema de secagem em coluna e camada fixa. O secador constitui-se de três colunas de secagem, com um sistema de cinco bandejas teladas móveis em cada coluna, nas quais foram realizados os controles da temperatura e umidade do produto. As temperaturas do ar de secagem foram de 40,60 e 80 ${ }^{\circ} \mathrm{C}$, controladas na entrada do secador, utilizando-se termômetro e termopares ligados a um termostato, para acionamento das resistências e manutenção da 
temperatura de secagem estabelecida. A temperatura e umidade relativa do ambiente foram registradas por um termo-higrógrafo instalado próximo à entrada de ar do ventilador. O fluxo de ar foi de $20 \mathrm{~m}^{3} \mathrm{~min}^{-1} \mathrm{~m}^{-2}$, registrado em anemômetro com precisão de $\pm 2 \%$, com leitura digital, na saída de ar do secador.

As amostras foram coletadas aleatoriamente, 24 horas antes do início de cada teste de secagem, determinado-se a umidade inicial, pelo método da estufa a $105^{\circ} \mathrm{C}$ por 24 horas (BRASIL, 1992). O valor do teor de água inicial foi o ponto de referência para o controle da secagem do produto. O teor de água, durante o período de secagem, foi obtido pela diferença de peso do produto nas bandejas. A pesagem das bandejas foi feita em intervalos de 30 minutos, até que o produto estivesse com uma umidade média de $13 \%$. Após a secagem, os grãos foram armazenados em embalagens de polietileno durante $0,2,4,6$ e 8 meses em câmara a $20 \pm 2{ }^{\circ} \mathrm{C}$.

A energia requerida para a secagem do trigo, nas diferentes condições, foi determinada pelo software PSIPROC desenvolvido por BIAGI e SILva (1990). Para o cálculo da energia requerida, foi usado um valor de pressão barométrica de 706,5 mmHg e valor de Kwat/ $\mathrm{h}$ de $\mathrm{R} \$ 0,30175$.

Para a obtenção da farinha, foi realizada a moagem de aproximadamente $1,5 \mathrm{~kg}$ de grãos, por amostra, em moinho de rolos Brabender, modelo Quadrumat Sênior. Após a secagem e, em todos os períodos de armazenamento, foram realizados no laboratório de cereais, raízes e tubérculos na Faculdade de Engenharia de Alimentos, da UNICAMP os seguintes testes de qualidade de grãos: peso hectolitro (PH) - determinado em Balança Dalle Molle (sistema Schoper), de acordo com as especificações do fabricante (Dalle Molle); número de queda (Falling Number) - realizado segundo método 56-81B da AACC (1995), utilizando-se aparelho "Falling Number" (Perten Instruments, Suécia); viscosidade - realizado segundo instruções do manual de operação para determinar o Rapid Visco Analyser (NewPort Scientific, 1995), considerando-se a viscosidade máxima, como o valor máximo na curva amilográfica; e propriedades de extensão da massa - realizado segundo o método 54-30A da AACC (1995), utilizando-se alveógrafo CHOPIN (Trippete \& Renauld, França), modelo 8120, acoplado de um RCV4 da mesma marca.

A análise estatística dos resultados dos diferentes tratamentos de secagem foi realizada utilizando-se o programa SAEG - Sistema para Análises Estatísticas, versão 8.0, desenvolvido pela Universidade Federal de Viçosa, e as médias foram comparadas pelo teste de Tukey ao nível de 5\%. Para os períodos de armazenagem, foram elaboradas curvas de tendências das médias dos tratamentos, utilizando-se modelo polinomial. Cada genótipo foi analisado separadamente, apresentando-se os dados das médias originais.

\section{RESULTADOS E DISCUSSÃO}

Na tabela 1, são apresentados os valores médios experimentais e calculados referentes ao processo de secagem estacionária, para cada temperatura do ar de secagem e teor de água inicial de grãos de trigo comum - IAC 24 e IAC 289 - e duro IAC 1003. Observa-se aumento da velocidade de secagem com o aumento da temperatura, sendo menor o tempo de exposição do produto ao ar aquecido para se atingir o teor de água final. Além do teor de água inicial do produto, fluxo de ar e temperatura de secagem, a variação das condições ambientais temperatura e umidade relativa - podem contribuir para a eficiência do processo de secagem.

A temperatura média da massa de grãos durante os diferentes tratamentos de secagem variou de 33,2 a $54,6^{\circ} \mathrm{C}$ para o IAC 24 (Tabela 1), de 32,9 a $55,2{ }^{\circ} \mathrm{C}$ para o IAC 289 e de 33,9 a $54,2{ }^{\circ} \mathrm{C}$ para o IAC 1003. A temperatura mais elevada do ar de secagem foi de $77,1^{\circ} \mathrm{C}$, para o IAC 24 .

Para valores diferentes do teor de água inicial do produto e da temperatura de secagem há uma variação no gradiente de umidade, que é a diferença entre os valores de umidade do trigo nos extremos da coluna de secagem. Diante disso, evidencia-se o problema na secagem estática do trigo, ou seja, uma secagem acentuada nas camadas inferiores e menor nas camadas superiores. Com o aumento da temperatura do ar de secagem e do teor de água inicial do produto há uma tendência para acréscimo do gradiente de umidade.

Com relação à energia requerida para aquecimento do ar de secagem, observa-se menor valor na secagem a $40^{\circ} \mathrm{C}$, porém nem sempre o menor custo, pois o tempo de secagem é bem maior, principalmente para a $1 .^{\mathrm{a}}$ colheita (Tabela 1 ), em que o menor custo foi com a secagem a $80{ }^{\circ} \mathrm{C}$, para o IAC 24 , e a $60{ }^{\circ} \mathrm{C}$ para o IAC 24 e IAC 289 , e a $40^{\circ} \mathrm{C}$ para o IAC 1003 na $2 .^{a}$ colheita.

Nos resultados de qualidade de grãos, podese observar uma diferença no peso hectolitro entre os genótipos e maiores valores para o genótipo IAC 1003 (Tabela 2). Os grãos de trigo duro são tipicamente maiores, mais pesados e mais duros que os de trigo comum, porém sua massa é menos elástica do que a do trigo comum, portanto, de qualidade inferior para a produção de pães, mas específica para massas alimentícias (IAC, 2002). 
Tabela 1: Valores médios experimentais e calculados dos parâmetros de secagem de trigo comum (IAC 24 e IAC 289) e trigo duro (IAC 1003) para a $1{ }^{\text {a }}$ e $2 .{ }^{\text {a colheitas }}$

\begin{tabular}{|c|c|c|c|c|c|c|c|c|c|c|c|c|c|c|c|c|c|c|}
\hline \multirow{3}{*}{ Parâmetros } & \multicolumn{6}{|c|}{ IAC 24} & \multicolumn{6}{|c|}{ IAC 289} & \multicolumn{6}{|c|}{ IAC 1003} \\
\hline & \multicolumn{3}{|l|}{ 1. ${ }^{\mathrm{a}} \mathrm{C}$} & \multicolumn{3}{|l|}{ 2. ${ }^{\mathrm{a}} \mathrm{C}$} & \multicolumn{3}{|l|}{ 1. ${ }^{\mathrm{a}} \mathrm{C}$} & \multicolumn{3}{|l|}{$2{ }^{\mathrm{a} C}$} & \multicolumn{3}{|l|}{$1^{\mathrm{a}} \mathrm{C}$} & \multicolumn{3}{|l|}{ 2. ${ }^{a} \mathrm{C}$} \\
\hline & $40^{\circ} \mathrm{C}$ & $60^{\circ} \mathrm{C}$ & $80^{\circ} \mathrm{C}$ & $40^{\circ} \mathrm{C}$ & $60^{\circ} \mathrm{C}$ & $80^{\circ} \mathrm{C}$ & $40^{\circ} \mathrm{C}$ & $60^{\circ} \mathrm{C}$ & $80^{\circ} \mathrm{C}$ & $40^{\circ} \mathrm{C}$ & $60^{\circ} \mathrm{C}$ & $80^{\circ} \mathrm{C}$ & $40^{\circ} \mathrm{C}$ & $60^{\circ} \mathrm{C}$ & $80^{\circ} \mathrm{C}$ & $40^{\circ} \mathrm{C}$ & $60^{\circ} \mathrm{C}$ & $80^{\circ} \mathrm{C}$ \\
\hline \multicolumn{19}{|c|}{ Valores Médios Experimentais } \\
\hline T. Secagem $\left({ }^{\circ} \mathrm{C}\right)$ & 39,8 & 58,3 & 76,6 & 39,9 & 58,4 & 77,1 & 39,3 & 56,3 & 75,2 & 39,6 & 57,6 & 76,2 & 39,7 & 57,9 & 75,5 & 40,1 & 57,7 & 76,5 \\
\hline T. do produto $\left({ }^{\circ} \mathrm{C}\right)$ & 34,1 & 45,4 & 54,6 & 33,2 & 45,4 & 52,3 & 32,9 & 43,9 & 53,7 & 33,8 & 46,2 & 55,2 & 33,9 & 45,3 & 54,2 & 34,4 & 44,2 & 54,0 \\
\hline T. ambiente $\left({ }^{\circ} \mathrm{C}\right)$ & 27,3 & 23,4 & 27,0 & 25,8 & 30,5 & 24,7 & 25,7 & 24,8 & 25,3 & 26,79 & 30,8 & 26,7 & 24,9 & 27,3 & 26,4 & 30,2 & 31,1 & 29,5 \\
\hline U. Relativa (\%) & 54,6 & 81,4 & 59,5 & 73,1 & 61,3 & 76,3 & 87,4 & 92,0 & 78,8 & 67,3 & 63,8 & 80,0 & 86,9 & 65,4 & 66,0 & 61,7 & 56,4 & 63,5 \\
\hline $\begin{array}{l}\text { Teor de água } \\
\text { inicial (\%) }\end{array}$ & 30,0 & 30,0 & 30,0 & 21,4 & 21,4 & 21,4 & 35,0 & 35,0 & 35,0 & 23,4 & 23,4 & 23,4 & 31,6 & 31,6 & 31,6 & 22,2 & 22,2 & 22,2 \\
\hline $\begin{array}{l}\text { Teor de água } \\
\text { final (\%) }\end{array}$ & 13,1 & 12,4 & 13,6 & 13,3 & 12,7 & 11,6 & 13,4 & 12,7 & 12,8 & 13,1 & 13,1 & 12,3 & 13,4 & 12,6 & 12,9 & 12,9 & 13,4 & 12,7 \\
\hline $\begin{array}{l}\text { Gradiente de } \\
\text { umidade (\%) }\end{array}$ & 6,2 & 13,8 & 16,9 & 4,2 & 6,5 & 9,9 & 7,9 & 21,6 & 21,8 & 3,9 & 7,4 & 10,7 & 9,1 & 15,1 & 19,2 & 3,8 & 7,6 & 11,7 \\
\hline $\begin{array}{l}\text { Tempo de } \\
\text { secagem (min) }\end{array}$ & 450 & 210 & 90 & 210 & 90 & 60 & 600 & 270 & 180 & 300 & 120 & 90 & 510 & 240 & 150 & 270 & 120 & 90 \\
\hline
\end{tabular}

Valores médios calculados

\begin{tabular}{|c|c|c|c|c|c|c|c|c|c|c|c|c|c|c|c|c|c|c|}
\hline Fluxo $\left(\mathrm{m}^{3} \cdot \mathrm{min}^{-1} \mathrm{~m}^{-2}\right)$ & 21,5 & 21,7 & 20,6 & 20,8 & 21,6 & 21,1 & 21,8 & 21,8 & 21,7 & 21,1 & 20,8 & 20,6 & 21,8 & 20,2 & 20,9 & 21,4 & 21,4 & 20,7 \\
\hline $\begin{array}{l}\text { Energia requerida } \\
(\mathrm{kJ} / \mathrm{min})\end{array}$ & 14,2 & 37,7 & 52,2 & 15,2 & 30,4 & 49,1 & 16,0 & 34,9 & 52,3 & 14,9 & 28,8 & 50,3 & 17,4 & 31,3 & 50,0 & 11,4 & 29,1 & 46,8 \\
\hline $\begin{array}{l}\text { Custo }(\mathrm{R} \$ / \mathrm{kg} \text { de } \\
\text { produto } \mathrm{seco})\end{array}$ & 0,06 & 0,07 & 0,04 & 0,03 & 0,02 & 0,02 & 0,09 & 0,09 & 0,09 & 0,04 & 0,03 & 0,04 & 0,08 & 0,07 & 0,07 & 0,02 & 0,03 & 0,04 \\
\hline $\begin{array}{l}\text { Custo }(\mathrm{R} \$ / \mathrm{t} \text {. de } \\
\text { produto seco) }\end{array}$ & 59,5 & 73,7 & 44,0 & 26,8 & 22,9 & 24,7 & 89,4 & 87,8 & 87,7 & 37,5 & 28,9 & 37,9 & 82,6 & 69,9 & 69,8 & 25,8 & 29,3 & 35,3 \\
\hline
\end{tabular}


Tabela 2. Valores médios de peso hectolitro para trigo comum (IAC24 e IAC289) e trigo duro (IAC1003), durante 8 meses de armazenagem

\begin{tabular}{|c|c|c|c|c|c|c|}
\hline \multirow{2}{*}{ Genótipos } & \multirow{2}{*}{ Tratamentos } & \multicolumn{5}{|c|}{ Armazenagem (meses) } \\
\hline & & 0 & 2 & 4 & 6 & 8 \\
\hline & & & & $\mathrm{kg} / \mathrm{Hl}$ & 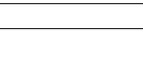 & 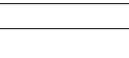 \\
\hline \multirow[t]{7}{*}{ IAC 24} & 1. ${ }^{\mathrm{a}} \mathrm{C} / 40^{\circ} \mathrm{C}$ & $75,4 \mathrm{~A}$ & $75,2 \mathrm{~A}$ & $75,7 \mathrm{~A}$ & $76,8 \mathrm{~A}$ & $76,5 \mathrm{~A}$ \\
\hline & $1 .^{\mathrm{a}} \mathrm{C} / 60^{\circ} \mathrm{C}$ & $73,8 \mathrm{D}$ & $73,0 \mathrm{D}$ & $72,9 \mathrm{D}$ & $74,2 \mathrm{CD}$ & $74,3 \mathrm{BC}$ \\
\hline & 1. ${ }^{\mathrm{a}} \mathrm{C} / 80^{\circ} \mathrm{C}$ & $71,4 \mathrm{E}$ & $72,8 \mathrm{D}$ & $70,9 \mathrm{E}$ & $72,4 \mathrm{E}$ & $72,1 \mathrm{~F}$ \\
\hline & 2. ${ }^{a} \mathrm{C} / 40^{\circ} \mathrm{C}$ & $73,9 \mathrm{CD}$ & $73,8 \mathrm{~B}$ & $73,7 \mathrm{~B}$ & $74,3 \mathrm{C}$ & $74,1 \mathrm{C}$ \\
\hline & 2. ${ }^{a} \mathrm{C} / 60^{\circ} \mathrm{C}$ & $73,9 \mathrm{CD}$ & $73,4 \mathrm{C}$ & $73,4 \mathrm{C}$ & $74,0 \mathrm{CD}$ & $73,8 \mathrm{D}$ \\
\hline & 2. ${ }^{\mathrm{a}} \mathrm{C} / 80^{\circ} \mathrm{C}$ & $74,1 \mathrm{C}$ & $73,0 \mathrm{D}$ & $73,4 \mathrm{C}$ & $73,9 \mathrm{D}$ & $73,4 \mathrm{E}$ \\
\hline & 3. ${ }^{\mathrm{a}} \mathrm{C}$ & $74,8 \mathrm{~B}$ & $73,9 \mathrm{~B}$ & $72,9 \mathrm{D}$ & $74,7 \mathrm{~B}$ & $74,5 \mathrm{~B}$ \\
\hline \multirow[t]{7}{*}{ IAC 289} & 1. ${ }^{\mathrm{a}} \mathrm{C} / 40^{\circ} \mathrm{C}$ & $70,5 \mathrm{DE}$ & $70,8 \mathrm{CD}$ & $72,6 \mathrm{~A}$ & $73,0 \mathrm{~B}$ & $72,1 \mathrm{~B}$ \\
\hline & $1 .^{\mathrm{a}} \mathrm{C} / 60^{\circ} \mathrm{C}$ & $70,7 \mathrm{CD}$ & $71,1 \mathrm{BC}$ & $72,0 \mathrm{C}$ & $73,8 \mathrm{~A}$ & $72,8 \mathrm{~A}$ \\
\hline & $1 .^{\mathrm{a}} \mathrm{C} / 80^{\circ} \mathrm{C}$ & $72,8 \mathrm{~A}$ & $71,4 \mathrm{AB}$ & $72,4 \mathrm{AB}$ & $72,7 \mathrm{~B}$ & $71,7 \mathrm{C}$ \\
\hline & $2 .^{\mathrm{a}} \mathrm{C} / 40^{\circ} \mathrm{C}$ & $72,8 \mathrm{~A}$ & $71,7 \mathrm{~A}$ & $72,6 \mathrm{~A}$ & $72,9 \mathrm{~B}$ & 72,3 B \\
\hline & 2. ${ }^{a} \mathrm{C} / 60^{\circ} \mathrm{C}$ & $72,2 \mathrm{~B}$ & $71,1 \mathrm{BC}$ & $72,1 \mathrm{BC}$ & $72,6 \mathrm{~B}$ & $72,4 \mathrm{~B}$ \\
\hline & 2. ${ }^{a} \mathrm{C} / 80^{\circ} \mathrm{C}$ & $70,9 \mathrm{C}$ & $70,7 \mathrm{D}$ & $71,8 \mathrm{C}$ & $71,9 \mathrm{C}$ & $71,4 \mathrm{C}$ \\
\hline & 3. ${ }^{\mathrm{a}} \mathrm{C}$ & $70,2 \mathrm{E}$ & $69,4 \mathrm{E}$ & $69,6 \mathrm{D}$ & $70,4 \mathrm{D}$ & $70,3 \mathrm{D}$ \\
\hline \multirow[t]{7}{*}{ IAC 1003} & 1. ${ }^{\mathrm{a}} \mathrm{C} / 40^{\circ} \mathrm{C}$ & $73,6 \mathrm{~F}$ & $74,3 \mathrm{D}$ & $73,9 \mathrm{D}$ & $73,2 \mathrm{E}$ & $68,9 \mathrm{~F}$ \\
\hline & $1 .^{\mathrm{a}} \mathrm{C} / 60^{\circ} \mathrm{C}$ & $74,0 \mathrm{E}$ & $74,0 \mathrm{D}$ & $74,0 \mathrm{D}$ & $74,1 \mathrm{D}$ & $69,5 \mathrm{E}$ \\
\hline & 1. ${ }^{\mathrm{a}} \mathrm{C} / 80^{\circ} \mathrm{C}$ & $74,7 \mathrm{D}$ & $73,2 \mathrm{E}$ & $73,4 \mathrm{E}$ & $73,1 \mathrm{E}$ & $70,8 \mathrm{D}$ \\
\hline & 2. ${ }^{a} \mathrm{C} / 40^{\circ} \mathrm{C}$ & $78,6 \mathrm{~B}$ & $76,4 \mathrm{C}$ & $76,9 \mathrm{C}$ & $78,2 \mathrm{~B}$ & $77,9 \mathrm{~B}$ \\
\hline & 2. ${ }^{\mathrm{a}} \mathrm{C} / 60^{\circ} \mathrm{C}$ & $78,0 \mathrm{C}$ & $76,6 \mathrm{C}$ & $78,1 \mathrm{~B}$ & $78,0 \mathrm{~B}$ & $77,7 \mathrm{~B}$ \\
\hline & 2. ${ }^{a} \mathrm{C} / 80^{\circ} \mathrm{C}$ & $78,1 \mathrm{C}$ & 77,6 B & $76,7 \mathrm{C}$ & $76,7 \mathrm{C}$ & $77,2 \mathrm{C}$ \\
\hline & $3^{\mathrm{a}} \mathrm{C}$ & $79,1 \mathrm{~A}$ & $78,4 \mathrm{~A}$ & $78,6 \mathrm{~A}$ & $79,2 \mathrm{~A}$ & $78,8 \mathrm{~A}$ \\
\hline
\end{tabular}

Médias seguidas de letras iguais, na mesma coluna, não diferem entre si pelo teste de Tukey a 5\%.

Para os genótipos IAC 24 e IAC 289, verificouse pequena variação entre os tratamentos de colheita e secagem (Tabela 2), apesar de essa ter sido significativa. Durante a armazenagem houve uma pequena variação dos valores de $\mathrm{PH}$, porém sem uma tendência (Figura 1A).

Os grãos do genótipo IAC 24 podem ser classificados como médios, ou seja, com valores de $\mathrm{PH}$ entre $72-75 \mathrm{~kg} / \mathrm{Hl}$ (WILLIANs et al., 1988), à exceção da 1. ${ }^{\mathrm{a}} \mathrm{C} / 80{ }^{\circ} \mathrm{C}$, quando são classificados como grãos leves. Para o genótipo IAC 289, os valores de $\mathrm{PH}$ podem ser classificados como leve e médio, obtendose os menores valores na $3 .^{\mathrm{a}}$ colheita.
No genótipo IAC 1003 constatou-se uma redução do PH com a armazenagem (Figura 1A). Nos tratamentos da $1^{\mathrm{a}}$ colheita os valores de $\mathrm{PH}$ foram inferiores aos da $2 .^{a}$ e $3 .^{a}$ colheitas (Tabela 2) e podem ser classificados como médios - entre $72-75 \mathrm{~kg} / \mathrm{Hl}$ e os da $2 .^{a}$ e $3 .^{a}$ colheitas como pesados - entre 76-79 $\mathrm{kg} / \mathrm{Hl}$, segundo WiLlians et al. (1988).

O peso hectolitro é um índice referente ao rendimento dos grãos em farinha ou sêmola, sendo será mais elevado quanto maior for o valor obtido. Existem valores mínimos desse índice estabelecidos para os trigos moles e duros, sendo aqueles para o segundo tipo mais elevados (MANDARINO, 1993). 

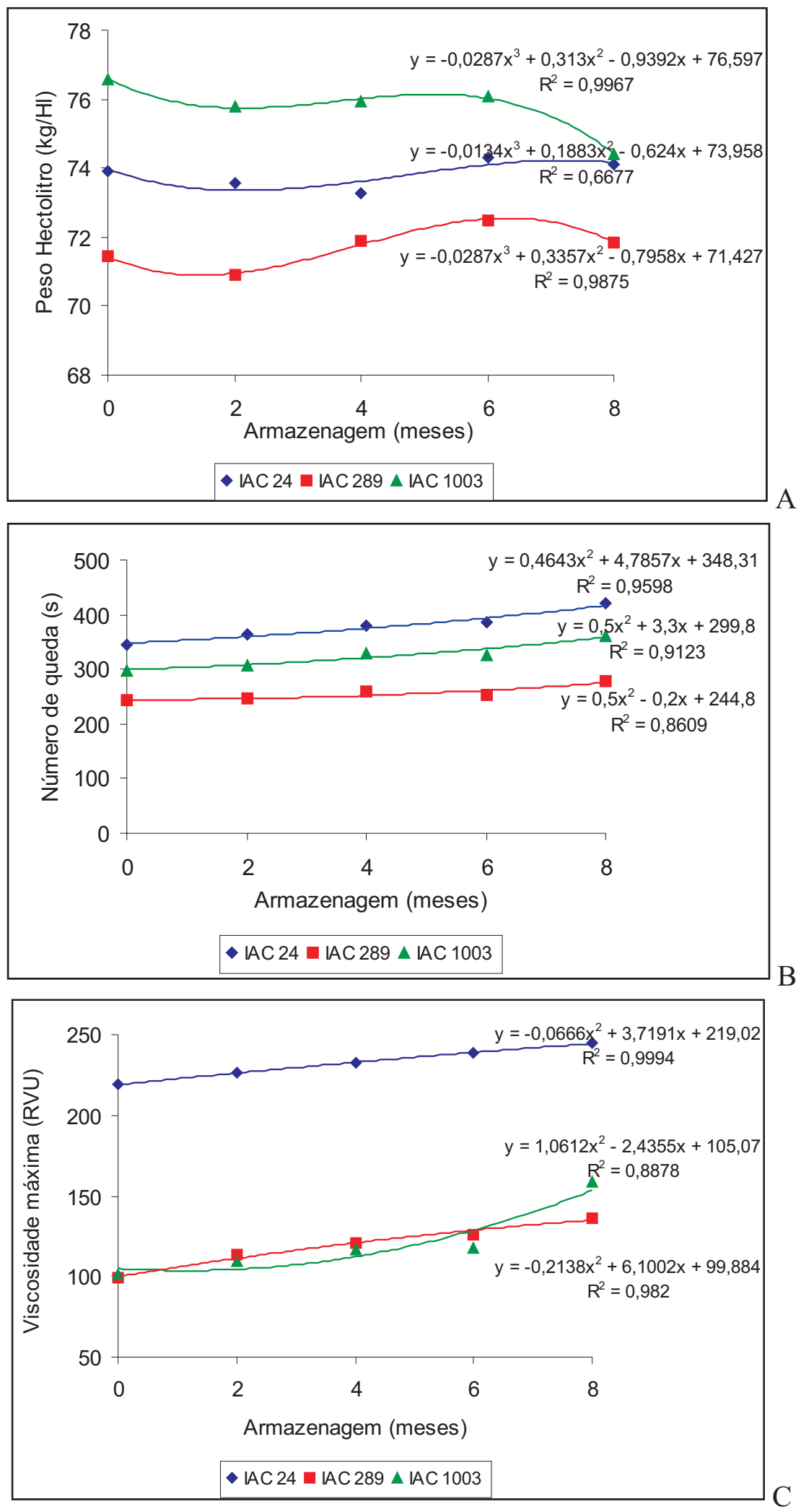

Figura 1: Curvas de tendência de Peso hectolitro (A), Número de queda (B) e Viscosidade máxima (C) para trigo comum (IAC 24 e IAC 289) e trigo duro (IAC 1003), durante oito meses de armazenagem. 
O fato de um genótipo de trigo com maior valor de PH não assegura que seja de melhor qualidade. Essa relação somente será significativa quando se comparar a mesma variedade com valores de $\mathrm{PH}$ bem diferenciados, pois muitos fatores são causas de erros na determinação desse índice, como por exemplo, os espaços vazios entre os grãos, o teor de água e o tipo e a quantidade de impurezas presentes na amostra (MANDARINO, 1993; Guarienti, 1996).

Segundo MAndarino (1993), o teste de PH é bastante utilizado na indústria, como teste rápido e indicativo da qualidade do grão recebido para moagem. No entanto, é difícil quantificar a qualidade de um determinado trigo para uso em panificação a partir desse resultado.

O número de queda - Falling Number - dos genótipos IAC 24, IAC 289 e IAC 1003, para os diferentes tratamentos, são apresentados na Tabela 3. Para o genótipo IAC 24, em praticamente todos os grãos colhidos com maior teor de água nas $1^{\mathrm{a}}$ e $2^{\mathrm{a}}$ colheitas, independentemente da temperatura de secagem, verificou-se pequena atividade da enzima alfa-amilase, com número de queda 3350 , ou seja, valores significativamente maiores que os da $3^{\text {a }}$ colheita. Para a $3^{\mathrm{a}}$ colheita, constatou-se atividade enzimática ideal na farinha, segundo Perten (1964), pois durante todo o período de armazenagem, os valores determinados para o número de queda foram entre 201 a 305.

Segundo GuARIENTI (1996), a baixa atividade enzimática não é um problema de difícil solução. Em geral, na formulação dos reforçadores ou melhoradores utilizados em panificação, têm-se enzimas alfa-amilásicas fúngicas, com a finalidade de correção dessa deficiência na farinha.

Nos grãos do genótipo IAC 289 , da $1^{\text {a }}$ colheita $\mathrm{e}$, independentemente da temperatura de secagem, o número de queda foi significativamente maior que os demais (Tabela 3), com variação entre 300 e 480, que são valores de atividade enzimática ideal e baixa. Já nos grãos da $2^{a}$ colheita, verificou-se elevada atividade enzimática, ou seja, valores de número de queda abaixo de 200, durante os oito meses de armazenagem; na $3^{\text {a }}$ colheita a redução do número de queda foi ainda maior.

Tabela 3: Valores médios de número de queda para trigo comum (IAC24 e IAC289) e trigo duro (IAC1003), durante 8 meses de armazenagem

\begin{tabular}{|c|c|c|c|c|c|c|}
\hline \multirow{2}{*}{ Genótipos } & \multirow{2}{*}{ Tratamentos } & \multicolumn{5}{|c|}{ Armazenagem (meses) } \\
\hline & & 0 & 2 & 4 & 6 & 8 \\
\hline & & \multicolumn{5}{|c|}{ Segundos } \\
\hline \multirow[t]{7}{*}{ IAC 24} & 1. ${ }^{\mathrm{a}} \mathrm{C} / 40^{\circ} \mathrm{C}$ & $377 \mathrm{~A}$ & $373 \mathrm{BC}$ & 379 АВ & $403 \mathrm{AB}$ & $465 \mathrm{~A}$ \\
\hline & $1 .^{\mathrm{a}} \mathrm{C} / 60^{\circ} \mathrm{C}$ & $376 \mathrm{~A}$ & $415 \mathrm{AB}$ & $425 \mathrm{AB}$ & $424 \mathrm{AB}$ & $471 \mathrm{~A}$ \\
\hline & $1 .^{\mathrm{a}} \mathrm{C} / 80^{\circ} \mathrm{C}$ & $406 \mathrm{~A}$ & $438 \mathrm{~A}$ & $451 \mathrm{~A}$ & 466 A & $429 \mathrm{~A}$ \\
\hline & 2. ${ }^{\mathrm{a}} \mathrm{C} / 40^{\circ} \mathrm{C}$ & $349 \mathrm{AB}$ & $344 \mathrm{C}$ & $363 \mathrm{BC}$ & $370 \mathrm{ABC}$ & $419 \mathrm{~A}$ \\
\hline & 2. ${ }^{\mathrm{a}} \mathrm{C} / 60^{\circ} \mathrm{C}$ & $326 \mathrm{AB}$ & $352 \mathrm{C}$ & $362 \mathrm{BC}$ & $383 \mathrm{ABC}$ & $425 \mathrm{~A}$ \\
\hline & 2. ${ }^{\mathrm{a}} \mathrm{C} / 80^{\circ} \mathrm{C}$ & $322 \mathrm{AB}$ & $356 \mathrm{BC}$ & $386 \mathrm{AB}$ & 359 BC & $419 \mathrm{~A}$ \\
\hline & 3. ${ }^{\mathrm{a}} \mathrm{C}$ & 269 B & $271 \mathrm{D}$ & 295 C & 294 C & 315 B \\
\hline \multirow[t]{7}{*}{ IAC 289} & 1. ${ }^{\mathrm{a}} \mathrm{C} / 40^{\circ} \mathrm{C}$ & $318 \mathrm{~B}$ & $371 \mathrm{~A}$ & $379 \mathrm{~A}$ & $330 \mathrm{~B}$ & $432 \mathrm{~A}$ \\
\hline & 1. ${ }^{\mathrm{a}} \mathrm{C} / 60^{\circ} \mathrm{C}$ & $304 \mathrm{~B}$ & $330 \mathrm{~B}$ & 347 B & $349 \mathrm{~B}$ & $346 \mathrm{~B}$ \\
\hline & $1^{\mathrm{a}} \mathrm{C} / 80^{\circ} \mathrm{C}$ & $439 \mathrm{~A}$ & $365 \mathrm{~A}$ & $391 \mathrm{~A}$ & $381 \mathrm{~A}$ & $434 \mathrm{~A}$ \\
\hline & 2. ${ }^{\mathrm{a}} \mathrm{C} / 40^{\circ} \mathrm{C}$ & $175 \mathrm{C}$ & $172 \mathrm{C}$ & $189 \mathrm{C}$ & $200 \mathrm{C}$ & $215 \mathrm{C}$ \\
\hline & 2. ${ }^{\mathrm{a}} \mathrm{C} / 60^{\circ} \mathrm{C}$ & $169 \mathrm{C}$ & $184 \mathrm{C}$ & $197 \mathrm{C}$ & $206 C$ & $192 \mathrm{C}$ \\
\hline & $2 .^{\mathrm{a}} \mathrm{C} / 80^{\circ} \mathrm{C}$ & $185 \mathrm{C}$ & $183 \mathrm{C}$ & $182 \mathrm{C}$ & $197 \mathrm{C}$ & $212 \mathrm{C}$ \\
\hline & 3. ${ }^{\mathrm{a}} \mathrm{C}$ & $122 \mathrm{D}$ & 119 D & $127 \mathrm{D}$ & $118 \mathrm{D}$ & $119 \mathrm{D}$ \\
\hline \multirow[t]{7}{*}{ IAC 1003} & 1. ${ }^{\mathrm{a}} \mathrm{C} / 40^{\circ} \mathrm{C}$ & $340 \mathrm{~A}$ & 315 BC & $329 \mathrm{~A}$ & $357 \mathrm{AB}$ & $380 \mathrm{AB}$ \\
\hline & $1 .^{\mathrm{a}} \mathrm{C} / 60^{\circ} \mathrm{C}$ & $279 \mathrm{~B}$ & 319 B & $344 \mathrm{~A}$ & $341 \mathrm{AB}$ & $350 \mathrm{~B}$ \\
\hline & $1 .^{\mathrm{a}} \mathrm{C} / 80^{\circ} \mathrm{C}$ & 297 B & $364 \mathrm{~A}$ & $354 \mathrm{~A}$ & 359 A & $395 \mathrm{~A}$ \\
\hline & 2. ${ }^{\mathrm{a}} \mathrm{C} / 40^{\circ} \mathrm{C}$ & 292 B & 308 BC & $328 \mathrm{~A}$ & $310 \mathrm{C}$ & $345 \mathrm{BC}$ \\
\hline & $2 .^{\mathrm{a}} \mathrm{C} / 60^{\circ} \mathrm{C}$ & 279 B & 306 BC & $350 \mathrm{~A}$ & 319 BC & 391A \\
\hline & 2. ${ }^{\mathrm{a}} \mathrm{C} / 80^{\circ} \mathrm{C}$ & $302 \mathrm{AB}$ & $278 \mathrm{CD}$ & $318 \mathrm{AB}$ & $340 \mathrm{AB}$ & $365 \mathrm{AB}$ \\
\hline & 3. ${ }^{\mathrm{a}} \mathrm{C}$ & $304 \mathrm{~A}$ & $259 \mathrm{D}$ & 287 B & $266 \mathrm{D}$ & $309 \mathrm{C}$ \\
\hline
\end{tabular}

Médias seguidas de letras iguais, na mesma coluna, não diferem entre si pelo teste de Tukey a 5\%. 
A elevada atividade enzimática é indicativa do início do processo de germinação, durante o qual é diminuído o teor de amido do grão, devido a sua transformação em glicídios; porém não há se acúmulo destes, que são utilizados em grande parte na respiração, para produção de energia, bem como na síntese de outras moléculas complexas (Popinigis, 1985). A elevada atividade enzimática só pode ser corrigida pela mescla de trigo ou de farinha complementares, visando-se à "diluição" do excesso de alfa-amilase.

No genótipo IAC 1003 e, em praticamente todos os tratamentos, a atividade enzimática foi ideal, ou seja, com valores de número de queda entre $201 \mathrm{e}$ 350 (Tabela 3), com exceção de $1 .{ }^{\mathrm{a}} \mathrm{C} / 80{ }^{\circ} \mathrm{C}$, onde se observou um aumento nos valores com o decorrer da armazenagem; também para $1 .{ }^{\mathrm{a}} \mathrm{C} / 40{ }^{\circ} \mathrm{C}$ e $2^{\mathrm{a}} \mathrm{C} / 60$ e $80^{\circ} \mathrm{C}$, com um aumento no final da armazenagem, o que não se verificou nos grãos da $3^{\mathrm{a}}$ colheita. Os menores valores do número de queda foram observados na farinha da $3^{\mathrm{a}}$ colheita.

Com o decorrer da armazenagem, observa-se uma tendência de aumento do número de queda (Figura 1B) para o trigo comum, IAC 24 e IAC 289, e o trigo duro, IAC 1003.

Pelo número de queda pode-se estimar a capacidade de fermentação da massa de determinada farinha pois, em grãos de trigos germinados prestes a germinarem a atividade amilásica é elevada, a massa é liquefeita, dificultando-se o processo industrial (MANDARINO, 1993).

Tendência semelhante foi observada nos resultados de viscosidade máxima para trigo comum - IAC 24 e IAC 289, e trigo duro - IAC 1003. Para os grãos da $3 .^{\text {a }}$ colheita do genótipo IAC 24 , os valores foram significativamente menores que os da $1 .^{a}$ e 2 a $^{a}$ colheitas, independentemente da temperatura de secagem (Tabela 4).

Nos tratamentos de colheita e secagem do genótipo IAC 289 houve uma redução significativa da viscosidade máxima da farinha com o atraso da colheita, ou seja, os maiores valores foram observados nos tratamentos da $1 .^{\mathrm{a}}$ colheita, e os menores nos da 3. ${ }^{\mathrm{a}}$ colheita (Tabela 4). Assim como no número de queda, os valores de viscosidade máxima são bem menores nas $2 .^{a}$ e $3 .^{a}$ colheitas, ou seja, com o atraso da colheita, há um aumento da atividade da alfaamilase. Segundo MANDARINo (1993), com a ação da enzima na desintegração das longas cadeias do amido, há uma tendência para diminuição da viscosidade da farinha.
Para os grãos de IAC 1003, assim como para os dos outros genótipos, os menores valores foram constatados nas farinhas da 3 . $^{\mathrm{a}}$ colheita, ou seja, na última colheita, obteve-se maior atividade da alfaamilase (Tabela 4), embora apenas no genótipo IAC 289 tenha sido obtido número de queda inferior ao da faixa ideal - 201 a 350.

Nos três genótipos avaliados observa-se uma tendência de aumento da viscosidade máxima com a armazenagem, que é maior no trigo duro - IAC 1003 (Figura 1C). O mesmo foi observado para o número de queda (Figura 1B), o que demonstra redução da atividade enzimática dos grãos de trigo comum, IAC 24 e IAC 289, e trigo duro, IAC 1003, com a armazenagem.

Os valores de $\mathrm{W}$ - força geral do glúten, e a relação P/L de trigo comum, IAC 24 e IAC 289, e duro, IAC 1003, durante os oito meses de armazenagem (Tabela 5), podem ser utilizados na classificação do trigo de uso industrial. Segundo Pena et al. (1998), o trigo forte e extensível, apresenta um endosperma duro a semiduro, e pode ser usado na panificação mecanizada e como melhorador de trigos com menor força de glúten; o trigo médio e extensível também possui um endosperma duro a semiduro, e pode ser usado na panificação manual e semimecanizada; o trigo fraco e extensível possui um endosperma suave e pode ser usado na fabricação de biscoitos, pizzas e pães artesanais; o trigo médio e tenaz - pouco extensível, possui endosperma duro a semiduro, e pode ser usado em pizzas e biscoitos e, o trigo forte e tenaz, possui endosperma duro e cristalino, podendo ser usado em massas alimentícias.

A farinha com valores de $\mathrm{P} / \mathrm{L}$ menores do que 0,60 pode ser considerada de glúten extensível; de 0,61 a 1,20, de glúten balanceado, e maior do que 1,21, de glúten tenaz (GUARIENTI, 1996).

Nos grãos do IAC 24, nota-se que, no início da armazenagem apenas a farinha da $3^{a}$ colheita é classificada como média, ou seja, com um valor de $\mathrm{W}$ menor que $200 \times 10^{-4} \mathrm{~J}$, enquanto nos demais tratamentos é classificada como média-forte, com valores de $\mathrm{W}$ entre $201-300 \times 10^{-4} \mathrm{~J}$ e forte, de 301- 400 x $10^{-4} \mathrm{~J}$, segundo Williams et al., (1988).

Com a armazenagem, observou-se um aumento na força geral de glúten (W) em todos os tratamentos, obtendo-se os maiores valores com a secagem a $80^{\circ} \mathrm{C}$, após oito meses. $\mathrm{O}$ mesmo padrão foi observado para $\mathrm{P} / \mathrm{L}$, à exceção da $2 .{ }^{\mathrm{a}} \mathrm{C} / 40{ }^{\circ} \mathrm{C}$, e os maiores valores de $\mathrm{P} / \mathrm{L}$ nos grãos secos a $80^{\circ} \mathrm{C}$, ou seja, com a secagem diminuiu-se a elasticidade da farinha. 
Tabela 4: Valores médios de viscosidade máxima para trigo comum (IAC24 e IAC289) e trigo duro (IAC1003), durante 8 meses de armazenagem

\begin{tabular}{|c|c|c|c|c|c|c|}
\hline \multirow{2}{*}{ Genótipos } & \multirow{2}{*}{ Tratamentos } & \multicolumn{5}{|c|}{ Armazenagem (meses) } \\
\hline & & 0 & 2 & 4 & 6 & 8 \\
\hline & & \multicolumn{5}{|c|}{ RVU } \\
\hline \multirow[t]{7}{*}{ IAC 24} & 1. ${ }^{\mathrm{a}} \mathrm{C} / 40^{\circ} \mathrm{C}$ & $227,5 \mathrm{C}$ & $237,3 \mathrm{C}$ & $238,4 \mathrm{BC}$ & $256,7 \mathrm{C}$ & $252,7 \mathrm{C}$ \\
\hline & 1. ${ }^{\mathrm{a}} \mathrm{C} / 60^{\circ} \mathrm{C}$ & $233,0 \mathrm{C}$ & $270,3 \mathrm{~B}$ & $271,2 \mathrm{~A}$ & $268,1 \mathrm{~B}$ & $274,5 \mathrm{~B}$ \\
\hline & 1. ${ }^{\mathrm{a}} \mathrm{C} / 80^{\circ} \mathrm{C}$ & $282,1 \mathrm{~A}$ & 305,9 A & $276,3 \mathrm{~A}$ & $283,3 \mathrm{~A}$ & $294,0 \mathrm{~A}$ \\
\hline & 2. ${ }^{\mathrm{a}} \mathrm{C} / 40^{\circ} \mathrm{C}$ & 244,5 B & $188,2 \mathrm{E}$ & $234,8 \mathrm{C}$ & $248,5 \mathrm{CD}$ & $230,8 \mathrm{D}$ \\
\hline & 2. ${ }^{\mathrm{a}} \mathrm{C} / 60^{\circ} \mathrm{C}$ & $226,5 \mathrm{C}$ & $219,2 \mathrm{D}$ & $225,4 \mathrm{D}$ & $247,3 \mathrm{D}$ & $255,9 \mathrm{C}$ \\
\hline & 2. ${ }^{\mathrm{a}} \mathrm{C} / 80^{\circ} \mathrm{C}$ & $198,7 \mathrm{D}$ & $233,3 \mathrm{C}$ & 246,2 B & $225,3 \mathrm{E}$ & $259,1 \mathrm{C}$ \\
\hline & $3 .^{a} \mathrm{C}$ & $119,9 \mathrm{E}$ & $130,9 \mathrm{~F}$ & $138,0 \mathrm{E}$ & $141,0 \mathrm{~F}$ & $145,7 \mathrm{E}$ \\
\hline \multirow[t]{7}{*}{ IAC 289} & 1. ${ }^{\mathrm{a}} \mathrm{C} / 40^{\circ} \mathrm{C}$ & $183,9 \mathrm{~A}$ & $188,7 \mathrm{~B}$ & 199,6 B & $180,4 \mathrm{C}$ & $323,0 \mathrm{~A}$ \\
\hline & 1. ${ }^{\mathrm{a}} \mathrm{C} / 60^{\circ} \mathrm{C}$ & 147,8 B & $162,3 \mathrm{C}$ & $177,9 \mathrm{C}$ & $188,4 \mathrm{~B}$ & $168,4 \mathrm{C}$ \\
\hline & 1. ${ }^{\mathrm{a}} \mathrm{C} / 80^{\circ} \mathrm{C}$ & $181,6 \mathrm{~A}$ & $208,4 \mathrm{~A}$ & $213,4 \mathrm{~A}$ & $236,3 \mathrm{~A}$ & $208,3 \mathrm{~B}$ \\
\hline & 2. ${ }^{\mathrm{a}} \mathrm{C} / 40^{\circ} \mathrm{C}$ & $40,1 \mathrm{E}$ & $60,0 \mathrm{E}$ & $75,9 \mathrm{D}$ & $85,4 \mathrm{D}$ & $76,1 \mathrm{D}$ \\
\hline & $2 \cdot{ }^{\mathrm{a}} \mathrm{C} / 60^{\circ} \mathrm{C}$ & $48,2 \mathrm{D}$ & $71,1 \mathrm{D}$ & $74,7 \mathrm{D}$ & $81,6 \mathrm{D}$ & $72,1 \mathrm{D}$ \\
\hline & 2. ${ }^{\mathrm{a}} \mathrm{C} / 80^{\circ} \mathrm{C}$ & $57,5 \mathrm{C}$ & $66,5 \mathrm{D}$ & $67,6 \mathrm{E}$ & $74,6 \mathrm{E}$ & $70,5 \mathrm{D}$ \\
\hline & 3. ${ }^{\mathrm{a}} \mathrm{C}$ & $32,1 \mathrm{~F}$ & $36,9 \mathrm{~F}$ & $39,0 \mathrm{~F}$ & $36,8 \mathrm{~F}$ & $35,3 \mathrm{E}$ \\
\hline \multirow[t]{7}{*}{ IAC 1003} & 1. ${ }^{\mathrm{a}} \mathrm{C} / 40^{\circ} \mathrm{C}$ & $104,2 \mathrm{C}$ & $113,2 \mathrm{C}$ & $111,4 \mathrm{C}$ & $122,8 \mathrm{~B}$ & $182,4 \mathrm{~B}$ \\
\hline & 1. ${ }^{\mathrm{a}} \mathrm{C} / 60^{\circ} \mathrm{C}$ & $91,1 \mathrm{E}$ & $123,2 \mathrm{~B}$ & $120,5 \mathrm{~B}$ & $112,2 \mathrm{C}$ & $183,3 \mathrm{~B}$ \\
\hline & 1. ${ }^{\mathrm{a}} \mathrm{C} / 80^{\circ} \mathrm{C}$ & $124,9 \mathrm{~A}$ & $138,7 \mathrm{~A}$ & $151,0 \mathrm{~A}$ & $141,5 \mathrm{~A}$ & $212,8 \mathrm{~A}$ \\
\hline & 2. ${ }^{\mathrm{a}} \mathrm{C} / 40^{\circ} \mathrm{C}$ & $96,8 \mathrm{D}$ & $108,7 \mathrm{CD}$ & 122,4 B & $115,1 \mathrm{C}$ & $130,4 \mathrm{E}$ \\
\hline & 2. ${ }^{a} \mathrm{C} / 60^{\circ} \mathrm{C}$ & $101,7 \mathrm{CD}$ & $105,0 \mathrm{D}$ & $125,5 \mathrm{~B}$ & 127,8 B & $142,7 \mathrm{D}$ \\
\hline & 2. ${ }^{\mathrm{a}} \mathrm{C} / 80^{\circ} \mathrm{C}$ & 118,9 B & $110,0 \mathrm{CD}$ & $110,5 \mathrm{C}$ & $127,8 \mathrm{~B}$ & $150,4 \mathrm{C}$ \\
\hline & 3. ${ }^{\mathrm{a}} \mathrm{C}$ & $73,4 \mathrm{~F}$ & $70,9 \mathrm{E}$ & $75,8 \mathrm{D}$ & $73,5 \mathrm{D}$ & $107,3 \mathrm{~F}$ \\
\hline
\end{tabular}

Médias seguidas de letras iguais, na mesma coluna, não diferem entre si pelo teste de Tukey a $5 \%$.

No genótipo IAC 289 e, apenas na $3 .^{\text {a }}$ colheita, o valor de $W$ foi menor que $200 \times 10^{-4} \mathrm{~J}$, caracterizando-se uma farinha de força de glúten média durante os oito meses de armazenagem nos demais tratamentos as farinhas foram força médiaforte, com W entre 201 a $300 \times 10^{-4} \mathrm{~J}$ (Tabela 5), à exceção da $1 .^{\mathrm{a}}$ e $2 .{ }^{\mathrm{a}} \mathrm{C} / 60{ }^{\circ} \mathrm{C}$, com força geral de glúten de 182,2 e $179,2 \times 10^{-4} \mathrm{~J}$, no início da armazenagem e aumento a partir do mês dois. Com relação aos valores $\mathrm{P} / \mathrm{L}$, observaram-se os maiores valores $-1,18-1,61$, no tratamento $1 .^{\mathrm{a}} \mathrm{C} / 80{ }^{\circ} \mathrm{C}$ e os menores $(<0,57)$ na $3 .^{\mathrm{a}}$ colheita.

Já no genótipo IAC 1003 não foi possível determinarem-se o W e o $\mathrm{P} / \mathrm{L}$ dos tratamentos 1 . $^{\mathrm{a}} \mathrm{e}$ 2. a. $\mathrm{C} / 80^{\circ} \mathrm{C}$, em qualquer mês de análise e a partir de seis meses de armazenagem, não houve determinação nos tratamentos, pois a massa formada por esta farinha era muito resistente, sendo ultrapassada a capacidade de leitura do equipamento (Tabela 5). Conforme o $\mathrm{W}$ determinado nos tratamentos, à exceção da $3 .^{\text {a }}$ colheita, a farinha é classificada como médiaforte, havendo um aumento na força de glúten com a armazenagem. Os valores de $\mathrm{P} / \mathrm{L}$ foram sempre superiores a 2,00, o que é uma característica da espécie, pois sua farinha é de muita resistência e pouca elasticidade, sendo ideal para massas alimentícias. Já o P/L detectado nos genótipos IAC 24 e IAC 289, com exceção do tratamento $1 .{ }^{\mathrm{a}} \mathrm{C} / 80{ }^{\circ} \mathrm{C}$, é indicativo de maior elasticidade da farinha, sendo ideal para panificação. 
Tabela 5: Valores alveográficos para W (x10 $\left.{ }^{-4} \mathrm{~J}\right)$ e P/L de trigo comum (IAC24 e IAC289) e trigo duro (IAC1003), durante 8 meses de armazenagem.

\begin{tabular}{|c|c|c|c|c|c|c|c|c|c|c|c|}
\hline \multirow{3}{*}{ Genótipos } & \multirow{3}{*}{ Tratamentos } & \multicolumn{10}{|c|}{ Armazenagem (meses) } \\
\hline & & \multicolumn{2}{|c|}{0} & \multicolumn{2}{|c|}{2} & \multicolumn{2}{|c|}{4} & \multicolumn{2}{|c|}{6} & \multicolumn{2}{|c|}{8} \\
\hline & & $\mathrm{W}$ & $\mathrm{P} / \mathrm{L}$ & W & $\mathrm{P} / \mathrm{L}$ & W & $\mathrm{P} / \mathrm{L}$ & $\mathrm{W}$ & $\mathrm{P} / \mathrm{L}$ & W & $\mathrm{P} / \mathrm{L}$ \\
\hline \multirow[t]{7}{*}{ IAC 24} & $1^{\mathrm{a}} \mathrm{C} / 40^{\circ} \mathrm{C}$ & 248,9 & 0,86 & 265,6 & 0,91 & 261,6 & 0,82 & 284,6 & 0,83 & 294,3 & 0,64 \\
\hline & $1^{\mathrm{a}} \mathrm{C} / 60^{\circ} \mathrm{C}$ & 289,4 & 0,71 & 253,7 & 1,12 & 327,0 & 0,89 & 302,6 & 0,82 & 323,1 & 1,18 \\
\hline & $1^{\mathrm{a}} \mathrm{C} / 80^{\circ} \mathrm{C}$ & 372,1 & 1,82 & 315,9 & 1,92 & 371,6 & 1,78 & 370,2 & 2,2 & 412,8 & 2,6 \\
\hline & $2^{\mathrm{a}} \mathrm{C} / 40^{\circ} \mathrm{C}$ & 302,8 & 0,78 & 267,8 & 069 & 338,8 & 1,13 & 352,4 & 0,78 & 342,0 & 0,66 \\
\hline & $2{ }^{\mathrm{a}} \mathrm{C} / 60^{\circ} \mathrm{C}$ & 317,8 & 0,84 & 308,0 & 0,83 & 317,2 & 0,88 & 328,9 & 0,91 & 356,3 & 1,19 \\
\hline & $2{ }^{\mathrm{a}} \mathrm{C} / 80^{\circ} \mathrm{C}$ & 292,3 & 0,91 & 299,1 & 0,95 & 352,5 & 1,19 & 347,0 & 1,17 & 427,1 & 1,26 \\
\hline & $3{ }^{\mathrm{a}} \mathrm{C}$ & 194,9 & 0,65 & 191,2 & 0,62 & 193,6 & 0,68 & 218,4 & 0,85 & 236,5 & 1,21 \\
\hline \multirow[t]{7}{*}{ IAC 289} & $1 .{ }^{\mathrm{a}} \mathrm{C} / 40^{\circ} \mathrm{C}$ & 247,9 & 0,90 & 238,7 & 0,88 & 251,8 & 0,90 & 230,2 & 1,19 & 266,2 & 1,08 \\
\hline & $1 .{ }^{\mathrm{a}} \mathrm{C} / 60^{\circ} \mathrm{C}$ & 182,2 & 1,83 & 180,5 & 0,65 & 244,2 & 0,93 & 247,0 & 0,95 & 247,2 & 0,88 \\
\hline & $1 .{ }^{\mathrm{a}} \mathrm{C} / 80^{\circ} \mathrm{C}$ & 296,3 & 1,40 & 283,8 & 1,61 & 296,9 & 1,51 & 295,6 & 1,18 & 328,3 & 1,37 \\
\hline & $2 .{ }^{\mathrm{a}} \mathrm{C} / 40^{\circ} \mathrm{C}$ & 213,2 & 0,60 & 228,2 & 0,51 & 232,2 & 0,62 & 260,4 & 0,67 & 258,3 & 0,67 \\
\hline & 2. ${ }^{\mathrm{a}} \mathrm{C} / 60^{\circ} \mathrm{C}$ & 179,2 & 0,83 & 231,4 & 0,61 & 228,2 & 0,63 & 265,6 & 0,58 & 243,3 & 0,67 \\
\hline & $2 .{ }^{\mathrm{a}} \mathrm{C} / 80^{\circ} \mathrm{C}$ & 228,9 & 0,54 & 229,2 & 0,70 & 265,5 & 0,91 & 241,5 & 0,77 & 238,1 & 0,85 \\
\hline & 3. ${ }^{\mathrm{a} C}$ & 139,9 & 0,49 & 162,2 & 0,36 & 240,3 & 0,38 & 195,3 & 0,34 & 187,0 & 0,57 \\
\hline \multirow[t]{7}{*}{ IAC 1003} & $1 .^{\mathrm{a}} \mathrm{C} / 40^{\circ} \mathrm{C}$ & 232,2 & 2,99 & 225,6 & 2,48 & 229,6 & 2,24 & - & - & - & - \\
\hline & $1 .{ }^{\mathrm{a}} \mathrm{C} / 60^{\circ} \mathrm{C}$ & 218,8 & 2,89 & 250,5 & 2,96 & - & - & - & - & - & - \\
\hline & $1 .{ }^{\mathrm{a}} \mathrm{C} / 80^{\circ} \mathrm{C}$ & - & - & - & - & - & - & - & - & - & - \\
\hline & $2 .{ }^{\mathrm{a}} \mathrm{C} / 40^{\circ} \mathrm{C}$ & 247,9 & 2,80 & 223,0 & 2,96 & - & - & - & - & - & - \\
\hline & $2 .{ }^{\mathrm{a}} \mathrm{C} / 60^{\circ} \mathrm{C}$ & 249,8 & 3,26 & 226,6 & 2,79 & - & - & - & - & - & - \\
\hline & $2 .{ }^{\mathrm{a}} \mathrm{C} / 80^{\circ} \mathrm{C}$ & - & - & - & - & - & - & - & - & - & - \\
\hline & 3. ${ }^{\mathrm{a} C}$ & 159,6 & 2,18 & 190,3 & 2,42 & 243,9 & 3,40 & - & - & - & - \\
\hline
\end{tabular}

Observando-se os resultados de número de queda, viscosidade e força geral de glúten para os genótipos IAC 24, IAC 289 e IAC 1003, nota-se uma melhora na farinha, em quase todos os tratamentos, com a armazenagem, que pode estar relacionada às modificações bioquímicas, com alteração da qualidade tecnológica do grão.

\section{CONCLUSÕES}

1. Não há redução na qualidade de grãos dos genótipos IAC 24 e IAC 1003 - peso hectolitro, número de queda, viscosidade e força geral de glúten, com a antecipação da colheita e secagem.

2. Há diminuição da atividade da alfaamilase do genótipo IAC 289, com a antecipação da colheita.
3. Com a armazenagem, há aumento da força geral de glúten e redução na atividade da alfa-amilase.

\section{AGRADECIMENTOS}

Os autores agradecem à FAPESP pelo auxílio à pesquisa, ao IAC pelo fornecimento dos grãos de trigo e ao CNPq pela concessão da bolsa de doutorado.

\section{REFERÊNCIAS BIBLIOGRÁFICAS}

AACC. AMERICAM ASSOCIATION OF CEREAL CHEMISTRS. Approved methods of AACC. 9. ed. St. Paul, v.1-2. 1995. 
BIAGI, J. D.; DALBELLO, O. Modelo psicrométrico para representar os processos de aquecimento, resfriamento, umidificação e mistura de dois ares. In: CONGRESSO BRASILEIRO DE ENGENHARIA AGRÍCOLA, 23, 1994, Campinas. Anais... SBEA, 1994. v.1.

BRASIL. Regras para Análise de Sementes. Ministério da Agricultura e Reforma Agrária. Brasília, 1992. 365p.

BRUM, P.A.R. O trigo na alimentação de aves. Avicultura Industrial, Porto Feliz, p.14-16, 2000.

CARVALHO, N. M.; NAKAGAWA, J. Sementes: Ciência, tecnologia e Produção. 4.ed. Jaboticabal: Funep, 2000. 588p.

DELOUCHE, J. C. Seed maturation. In: HANDBOOK OF SEED TECHNOLOGY. State College: Mississippi State University, 1971. p.17-21.

FNP - CONSULTORIA E COMÉRCIO. Disponível em: http:www.fnp.com.br. Acesso em: 14/7/2003.

GERMANI, R.; WATANABE, E.; CARVALHO, J. L. V. de; BENASSI, V. de T. Curso de controle de qualidade tecnológica do grão e da farinha de trigo. Rio de Janeiro: EMBRAPACTAA, 1998. 66p. (Apostila de curso).

GUARIENTI, E. Qualidade industrial de trigo. 2.ed. Passo Fundo: EMBRAPA-CNPT, 1996.36p.

HARRINGTON, J.F. Seed storage and longevity. In: KOSLOWSKI, T.T. Seed Biology. New York: Academic Press, v.3, p.145-245. 1972.
IAC. Recomendações da Comissão Técnica de Trigo para 2002. 3.ed. Campinas: Instituto Agronômico, 2002. 92p. (Boletim técnico, 167)

MANDARINO, J.M.G. Aspectos importantes para a qualidade do trigo. Londrina: EMBRAPA-CNPSo, 1993. 31p.

MARTINS, R.R.; FRANCO, J.B.R.; OLIVEIRA, P.A.V. Tecnologia de secagem de grãos. Passo Fundo: Embrapa Trigo/EMATER/ RS, 1999. 90p. (Embrapa Trigo. Documentos, 8)

NEWPORT SCIENTIFIC. Operation manual for the series 3. Rapid Visco Analyser. Austrália,1995. 92p.

PENA, R. J.; ORTIZ-MONASTERIO, J. I.;SAYRE, K. D. Estrategias para mejorar (o mantener) la calidad panadera em trigo de alto potencial de rendimiento. In: KOHLI, M. M.; MARTINO, D. Explorando altos rendimientos de trigo. Montevidéu: Uruguai: CIMMYT-INIA, 1998. p.289-306.

PERTEN, H. Application of the falling number method for evaluating alpha-amylase activity. Cereal Chemistry, St. Paul, v.41, n.3, p.127-140. 1964.

POPINIGIS, F. Fisiologia da semente. 2.ed. Brasília: AGIPLAN, 1985. 289p.

TOLEDO, F.F.; MARCOS FILHO, J. Manual das sementes: Tecnologia da produção. São Paulo: Agronômica Ceres, 1977. 224p.

WILLIANS, P.; EL-HARAMEIN, F. J.; NAKKOUL, H.; RIHAWI, $S$. Crop quality evaluation methods and guidelines. 2.ed. Aleppo, Syria: ICARDA, 1988. 145p. 